\title{
A "Double" Third Window Syndrome: The Case of Semicircular Canal Dehiscence in Twin Sisters
}

\author{
Serena Cocca' \\ Giulia Mignacco ${ }^{2}$ \\ Marco Mandalà ${ }^{2}$ \\ Caterina Giannitto ${ }^{3}$ \\ Andrea Alessandro \\ Esposito ${ }^{4}{ }^{4}$ \\ Simone Porcino ${ }^{2}$ \\ 'Department of Ear Nose Throat, \\ Ospedale Misericordia, Grosseto, GR, \\ 58100, Italy; ${ }^{2}$ Department of Ear Nose \\ Throat, AOUS "S.Maria alle Scotte"- \\ University Hospital of Siena, Siena, SI, \\ 53100, Italy; ${ }^{3}$ Department of Radiology, \\ Istituto Clinico Humanitas, Rozzano, MI, \\ 20089, Italy; ${ }^{4}$ Department of Radiology, \\ ASST Bergamo Ovest- Ospedale \\ "Treviglio-Caravaggio", Treviglio, BG, \\ 24047, Italy
}

\begin{abstract}
Third window lesions are defined as abnormal communications of the inner ear with adjacent spaces, namely the middle ear or cranial cavity. These lesions can occur at multiple anatomic locations, including the superior, posterior, and lateral semicircular canals; vestibule and vestibular aqueduct; and scala vestibuli of the cochlea. Semicircular canal dehiscence (SCD) is the most common condition wherein the temporal bone adjacent to the vestibular apparatus thins or is interrupted completely. Normally, there are two mobile windows: the oval window and round window. SCD results in a third mobile window in the inner ear, which causes an abnormal transmission of acoustic energy ("third-window effect") towards the vestibular end-organs and causes symptoms. Superior SCD is a welldescribed pathological condition with potentially debilitating symptoms, instead the dehiscence of the posterior canal is very rare. The diagnosis is difficult and often delayed because the symptoms can be absent or aspecific and confused with other diseases: vestibular disorders, cerebrovascular diseases, trauma, and tumors. We report a rare case about a "double" third window syndrome of twin sisters due to a bone defect of two different semicircular canals. A 34-year-old woman presented to the Otology Department of University Hospital with a worsening of tinnitus, autophony and vertigo. She had been treated for 3 years as if affected to Menière disease. An audiometric exam revealed a right mixed hearing loss with 20-40 dB air bone gap from 250 to $4000 \mathrm{~Hz}$. Vestibular examinations are unchanged compared to the past exams. But Valsalva maneuver induced pathological oscillopsia, vertigo and torsional up-beating nystagmus. Clinical manifestations may be like other diseases, such as Menière disease or perilymphatic fistula, confounding the diagnosis. Moreover, the appearance of the same symptoms in the patient's twin sister, confirmed by the radiological investigation, suggested the familiarity for semicircular canal dehiscence. Audiometric and vestibular examinations are important even if diagnosis is impossible without radiological investigation. Indeed, we describe the history, clinical profile and management of twin sisters who had similar symptoms (worsening vertigo induced by pressure, autophony, tinnitus and hearing loss) and different bony labyrinth defects. Surgical management resulted in complete resolution in imbalance 3 months after the surgery. This surprising case of homozygous twin sisters suggests that there may be a genetic aspect to the disease.
\end{abstract}

Keywords: vertigo, tinnitus, semicircular canals, hearing loss, dehiscence, third window

\section{Introduction}

Third window abnormalities are bony defects of the inner ear that enable abnormal communication with the middle ear and/or cranial cavity. Sound conduction relies on the presence of two physiologic windows between the fluid-filled inner ear and air-filled middle ear. 
Third window lesions can occur at multiple anatomic locations, including the superior, posterior, and lateral semicircular canals; vestibule and vestibular aqueduct; and scala vestibuli of the cochlea.

The most common example is semicircular canal dehiscence (SCD): it refers to extreme thinning and/or loss of the bony roof of the semicircular canal. The condition is idiopathic, although both congenital and acquired causes have been proposed: inflammation, neoplasia, surgery, congenital malformations, bone rarefaction/underpneumatization, barotrauma or direct mechanical trauma, and cerebrospinal fluid (CSF)/vascular pulsations (highriding jugular bulb).

The most common SCD is superior semicircular canal dehiscence (SSCD): it was first identified by Minor in $1998^{1}$ and consists of a defect of the upper external bone wall of the canal with resultant exposure of the membranous labyrinth to intracranial cavity. SCD usually may involve lateral canal representing the sequelae of advanced chronic otitis.

Posterior semi-circular canal dehiscence (PSCD) is a much rarer entity $(0.5 \%)$ but it can be observed in isolation or in combination with SSCD. The posterior semicircular canal can dehisce into the posterior fossa dura via a bony defect, or through communication with a high-riding jugular bulb.

Cochlear dehiscence refers to a deficiency of bone overlying the cochlea. In order for third window mechanics to occur, the scala vestibuli side of the cochlea must be involved.

Defects in the bony labyrinth enable dissipation of acoustic energy away from the cochleovestibular system into middle ear, dura mater, and/or vascular structures, altering perceptions of sound and balance. ${ }^{2}$ Clinically, patients with third window pathology present with stereotypical symptoms of vestibular activation, including directional vertigo, nystagmus, oscillopsia, dizziness, imbalance, and/or nausea. The symptoms may change or worsen over the time, confounding the diagnosis., ${ }^{3,4}$ Patients with SSCD typically present vertigo and oscillopsia induced by loud noises, such as traffic or shouting (Tullio phenomenon), by changes in pressure in the external ear canal or by Valsalva manoeuvres, as can occur with nose-blowing, or lifting of heavy objects (Hennebert sign) and nystagmus in vertical-torsional plane. ${ }^{5,6}$ Other symptoms of SCD are conductive hearing loss secondary to a "facilitated" bone conduction, autophony produced by dural oscillation, pulsatile tinnitus due to vascular vibrations, aural fullness. ${ }^{4,7,8}$ Symptoms and signs associated with a defect involving posterior semicircular canal has been recently described and they are most likely associated with a high-riding jugular bulb and fibrous dysplasia. $^{9-13}$

While SSCD has been well-described in the literature, there are few cases of PSCD reported. We present a rare case of PSCD that caused floating and "pseudo-conductive" hearing loss and vertigo in a young woman. Due to their nuanced characteristics, the symptoms were for a long time confused and treated as Menière disease. Obviously, the co-occurrence of SCD in both twin sisters strengthens the hypothesis that genetic factors may play a key role.

\section{Case Description}

A 34-year-old Caucasian female presented to the Otology Department of University Hospital for a worsening of tinnitus, autophony and vertigo.

She developed the first symptoms about three years before (hearing loss, aural fullness, persistent tinnitus in right ear, disequilibrium, and vertigo) and she was treated as having Menière disease. The symptoms were aggravated by a variation of atmospheric pressure and loud sounds. Vertigo and tinnitus could also be induced by the Valsalva maneuver. There was no history of cranial trauma.

When she came to the hospital, clinical examination revealed normal eardrums bilaterally. No evidence of spontaneous and positional nystagmus. Video-head impulse test and caloric responses were normal. Pure tone audiometry showed right mixed hearing loss with 20-40 dB air bone gap from 250 to $4000 \mathrm{~Hz}$ (Figure 1). The tympanometry findings were normal on both sides. Valsalva maneuver induced oscillopsia, vertigo and torsional upbeating nystagmus. cVEMP (cervical vestibular evoked myogenic potential) responses to air conduction stimulation at $500 \mathrm{~Hz}$ clicks were recorded with surface electrodes over the ipsilateral sternocleidomastoid muscle and base of the neck with the same ground electrode on the midline forehead. Patients are asked to turn their head in the opposite direction of the tested ear to flex the ipsilateral sternocleidomastoid muscle. cVEMP testing revealed a lowered threshold and an increased amplitude on the right side compared to the left (Figure 2). High resolution temporal bone computed tomography showed a high right jugular bulb which caused an area of PSCD. No dehiscence was evident on the superior canal or on either side (Figure 3). 
A

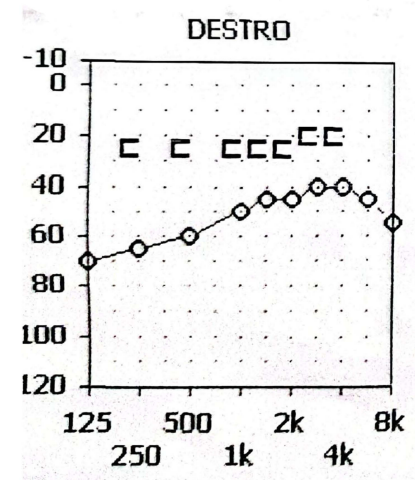

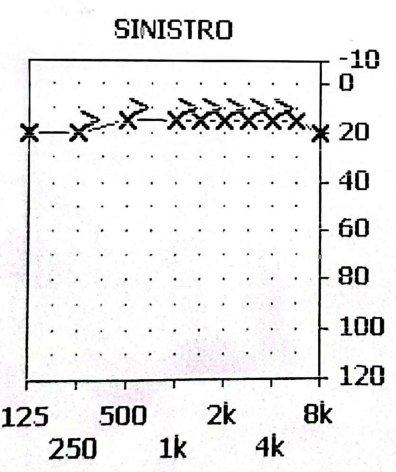
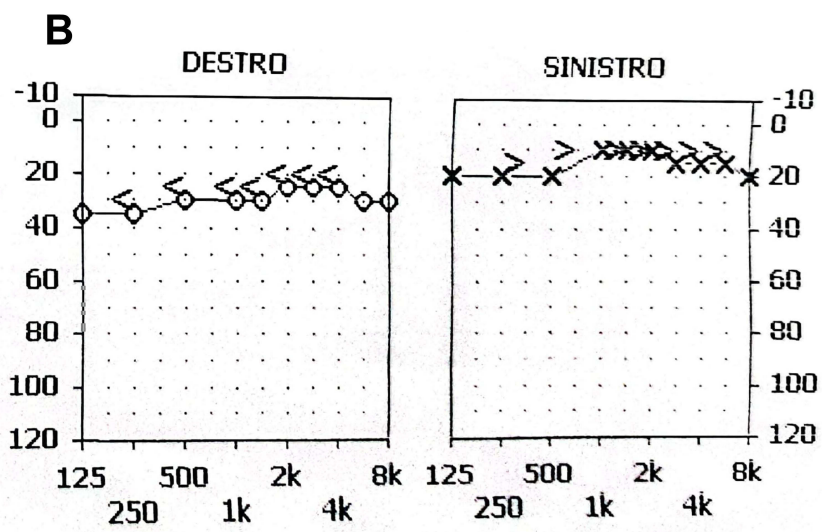

Figure I Conductive hearing loss on the right side. (A) Preoperative audiogram. (B) Postoperative audiogram after 2 months.
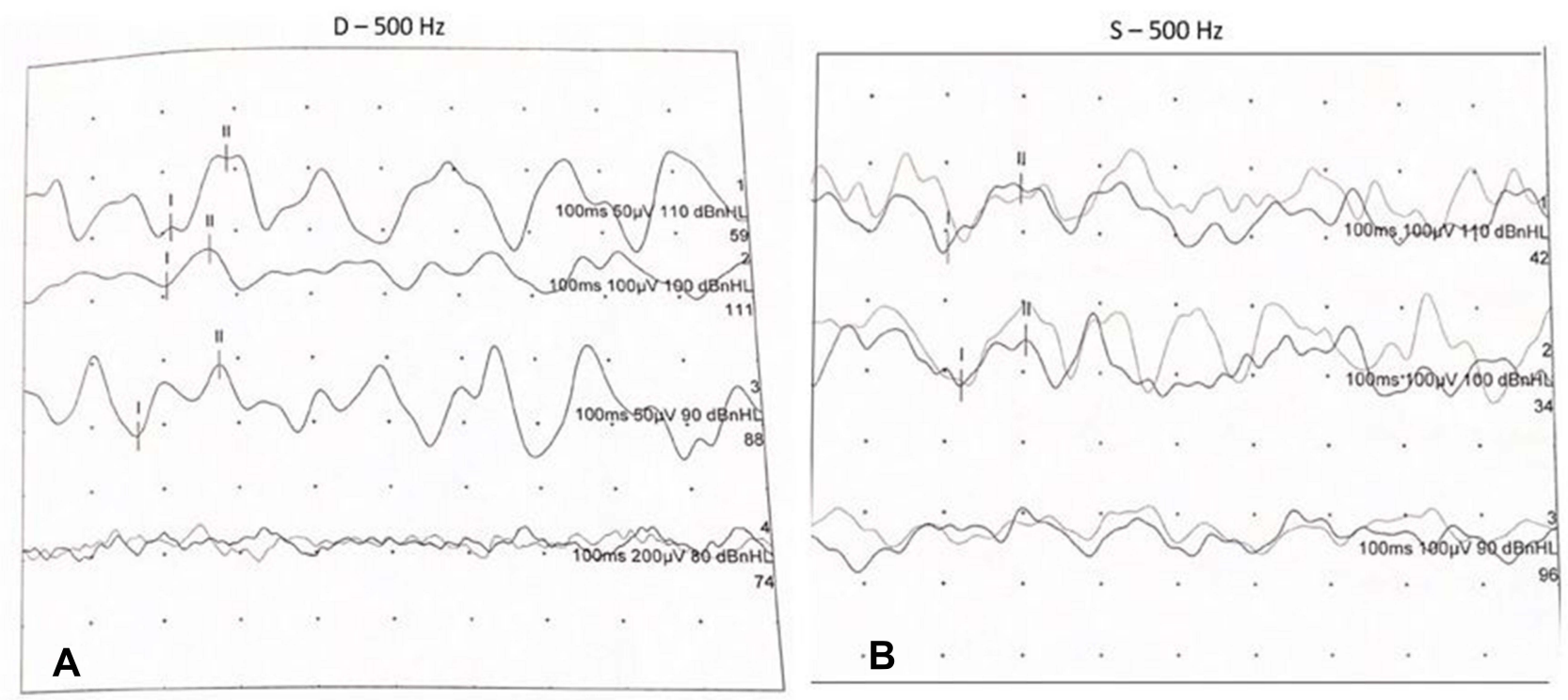

Figure 2 Cervical vestibular evoked myogenic potential (VEMPc) threshold test. Right side pre-operative test (A). Left side pre-operative test (B).

Due to continued daily discomfort, mostly noiseinduced vertigo that interfered with her work and life, the young patient underwent surgical intervention two weeks after the diagnosis.

After explaining about the possibilities of further hearing loss, facial paresis and persistence of vestibular symptoms, the patient signed a written consent to surgical procedures and to the use of data and images for teaching or research purposes. The posterior semicircular canal was approached through the mastoid bone. A transmastoid approach was taken to allow the decompression of the high-riding jugular bulb. The jugular bulb was decompressed using bipolar cautery and bone wax. The dehiscent posterior semicircular canal was plugged with muscle and autologous cartilage.
The patient's hospital course was uncomplicated, and she was discharged on the first postoperative day. On clinical follow up, three months after surgery, the patient described the resolution of loud sounds-induced vertigo, dizziness induced by a variation of atmospheric pressure and significant improvement of the tinnitus. Post-operative audiometry showed normal discrimination scores, resolution of the air-bone gap and pure tone average unchanged from the preoperative exam. c-VEMPs revealed an increased threshold and a decreased amplitude.

After about three months from the recovery, the patient's twin sister also started to complain of intermittent vertigo, autophony, hearing loss and mild tinnitus of the left ear. Auditory and vestibular features to those seen in 

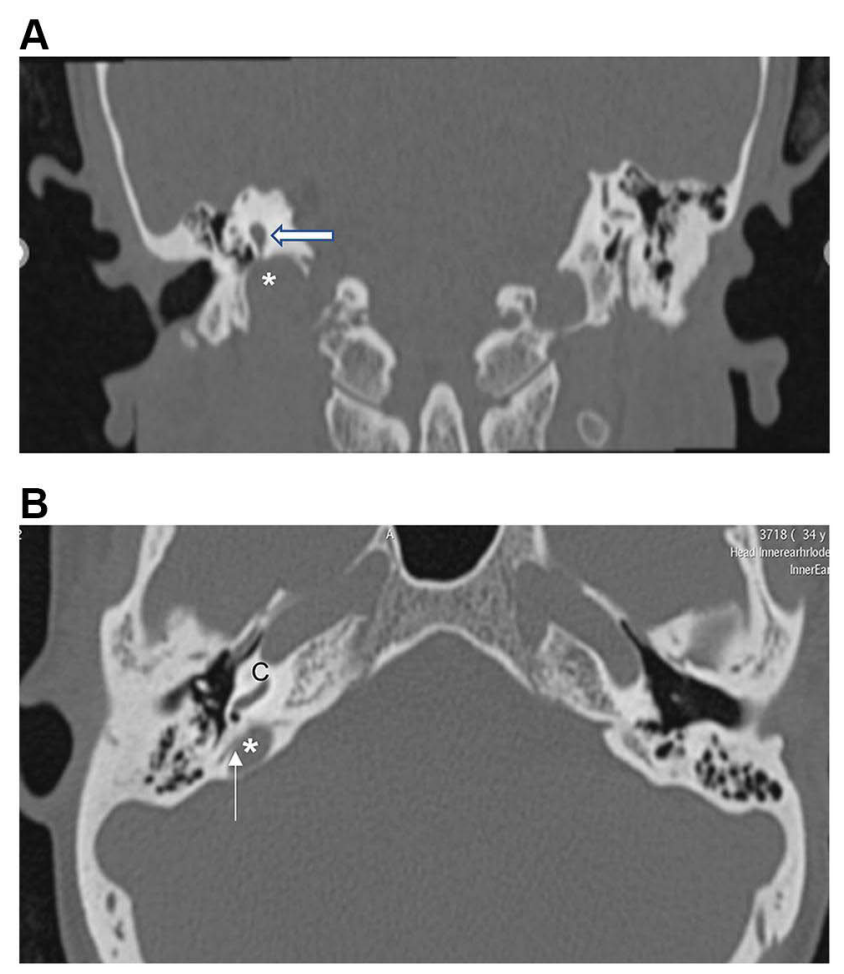

Figure 3 Computed tomography (CT) of the temporal bone (thickness $0.75 \mathrm{~mm}$ ). Coronal CT image confirms the absence of a bony separation of the right jugular bulb (asterisk) and the posterior semicircular canal (arrow) $(C=$ cochlea). (A) Axial cuts showing dehiscence between the the jugular bulb (asterisk) and the posterior semicircular canal (arrow) (B).

her sister led her ENT specialist to request audiological, vestibular, and radiological examinations (CT scan). And surprisingly the patient had SSCD even if the symptoms were less pronounced than her sister's. An updated audiometric assessment showed significant mixed hearing loss in the left ear. The diagnosis of SSCD has been confirmed by cVEMP registration that shows a pathologically increased amplitude and a decreased threshold. Watchful waiting is the solution because the symptoms do not compromise their quality of life, unlike the aforementioned first sister.

\section{Discussion}

Semicircular canal dehiscence syndrome has been increasingly recognized since the description by Minor et al, ${ }^{1}$ who reported on dehiscence of the SSCD. It is also referred to as third window syndrome (TWS), which consists of sound- and/or pressure-induced vertigo, oscillopsia, or disequilibrium. ${ }^{3,11}$ Diagnoses of TWS are aided by vestibular evoked myogenic potential (VEMP) testing, which might show reduced cervical VEMP (cVEMP) thresholds or increased ocular VEMP (oVEMP) amplitudes.

Absent bone separating the superior semicircular canal from the middle fossa on computed tomography (CT) corroborates the diagnosis. ${ }^{14}$

The aetiology of SCD is not understood entirely. Each canal has its own triggering or favouring factors. Lateral SCD has been mostly caused by chronic otitis media and cholesteatoma or surgical procedures such as mastoidectomy. SSCD is associated with increased intracranial pressure. A high-riding jugular bulb is the most common cause of PSCD, the other ones are fibrous dysplasia and apex cholesteatoma.11 PSCD is rarer than SSCD, but the precise incidence remains unknown. ${ }^{12,13}$ Congenital and acquired events may contribute to SCD: one hypothesis is that patients are born with thin or absent overlying of the superior semicircular canal and a "second event" (eg, skull base trauma, a Valsalva maneuver, intense acoustic exposure) causes an abrupt injury to the arcuate eminence. Another hypothesis is that dural pulsations over the arcuate eminence result in progressive loss of bone over the superior canal. ${ }^{15-17}$

In a recent systematic review, Lee et $\mathrm{al}^{18}$ analyzed the incidence of PSCD in the general population and summarized different PSCD, including alternatives to the classic TWS. PSCD is a rare phenomenon that might also present with dizziness and hearing loss inconsistent with thirdwindow symptomatology. In this review, two hundred and five studies were found, and 58 studies were included. In the 47 total patients, sound-induced vertigo, mixed hearing loss, and tinnitus were the most common presenting symptom. The incidence in pediatric patients ranged from $1.3 \%$ to $43 \%$. Jugular bulb abnormalities were common. In this study, ${ }^{18}$ the authors reported a case series about patients with PSCD diagnosed on imaging: only one patient had typical TWS and supra-threshold hearing, and four without TWS who experienced dizziness and hearing loss (HL).

Erdogan et $\mathrm{al}^{19}$ examined the CT scan of 410 adult patients with middle ear pathology (otitis media, cholesteatoma, etc.) and symptoms unrelated to the inner ear: only four patients (prevalence 1.2\%) had PSCD, three of whom demonstrated bilateral dehiscence.

While less common than SSCD, PSCD is reported to have a similar TWS complex, but can also be asymptomatic. $^{18}$

The clinical presentation can be mistaken for more common otological pathologies; therefore, the diagnosis 
is difficult and delayed as in our case. For example, the hearing loss is generally conductive, or less frequently sensorineural or mixed, and if associated with vertigo, fullness and tinnitus, the clinical presentation is very similar to Menière disease. Also in our case, the patient was treated for a long time as having Meniere disease. But analysing the clinical history of the patient, the intermittent course of her symptoms (aural fullness, tinnitus in right ear, disequilibrium, and vertigo) was often triggered to atmospheric pressure variations: even a simple plane flight was enough to trigger or worsen the symptoms. In the last period, the patient had become intolerant to loud sounds and even daily actions (for example, lifting a weight, taking a plane, going to high altitude) had become disabling. It is therefore no coincidence that the Valsalva maneuver induced oscillopsia, vertigo and up-beating nystagmus: none of these signs are typical of Menière disease. Therefore, clinical history and neurological evaluation are mandatory for diagnosis.

When conductive hearing loss is associated to dizziness, third-window lesions should be considered in the differential diagnosis especially in cases of patients with intact tympanic membrane and healthy middle ear. VEMP helps to distinguish the air-bone gap due to the ossicular pathology from that due to dehiscence of the canal: in the case of SCD there is a diminished threshold in $\mathrm{dB}$ and a greater amplitude of the muscular contraction. Furthermore, the stapedial reflex is normal in the case of $\mathrm{SCD}$, instead it is absent or altered in cases of middle ear pathology.

SCD signs and symptoms in members of the same family were similar. In our report, the first twin sister presented with more severe symptoms (hearing loss, aural fullness, persistent tinnitus in right ear, disequilibrium, and vertigo) which were aggravated by a variation of atmospheric pressure and loud sounds. Her twin sister had started experiencing the same symptoms but in a milder form and this led to suspicion of SCD. The finding of a bone defect of the semicircular canals in both twin sisters is suggestive.

Probably, it could be not entirely coincidental that twin sisters have different intensity of symptoms as two different semicircular canals are involved. The twin sisters have similar skull base topography: the first sister has a riding jugular bulb and posterior SCD, the second has superior SCD.

Niesten et $\mathrm{al}^{20}$ observed that skull base tomography and anatomic SCD defects were similar among first-degree relatives but all patients share the dehiscence or "near dehiscence" of the same semicircular canal, the superior canal. The authors of a case series suggested another interesting observation: symptoms seemed to be more pronounced in older patients (mothers) compared with their younger counterparts (daughters). Similarly, the sisters in our case also developed their symptoms almost simultaneously, probably because they are twin sisters.

In a retrospective case series, Heidenreich et al included 7 cases of superior semicircular canal dehiscence across 3 different families, one of which consisted of monozygotic adult twins, each of whom was diagnosed with unilateral superior SCD. ${ }^{21}$ This series is differentiated from prior reports of familial superior SCD because it lent additional support to a developmental etiology. Heidenreich refutes the hypothesis of Shutt et $\mathrm{al}^{22}$ according to whom patients with radiographic superior canal dehiscence were more likely to have had a higher mean BMI (calculated as weight in kilograms divided by height in meters squared). ${ }^{21}$ Obesity alone does not explain the occurrence of superior canal dehiscence syndrome. But, the presence of symptomatic SCD among first-degree relatives and similar skull base topography suggests that genetics may play a role in the etiology of SCD.

Undoubtedly, in our report the co-occurrence of SCD in both twin sisters reinforces the hypothesis that genetic factors may play a key role. However, it would be very interesting to study the two sisters from a genetic point of view to evaluate the cochlin $(\mathrm{COCH})$ gene mutation (C-to$\mathrm{T}$ base change in exon 3 at the DFNA9 locus) or any DFNA9 mutations. $\mathrm{COCH}$ is the most highly expressed protein in the inner ear and may be responsible for either structural integrity or antimicrobial activity. ${ }^{20}$

Obviously, the diagnostic confirmation is radiological since the conductive hearing loss in patients with intact tympanum could mimic otosclerosis or other middle ear diseases. Temporal computed tomography (CT) scans and magnetic resonance (MR) confirm the bone dehiscence overlying. CT is of great importance in the exclusion of other diseases, prevention of unnecessary surgeries such as stapedectomy and ossiculoplasty and evaluation of temporal region anatomy, defect size, and localization if surgery is planned. ${ }^{23}$ However, because not all SCD cases are symptomatic, it is recommended that the diagnosis should be made by evaluation of physical examination and audiological tests together with cross-sectional imaging. ${ }^{24}$

On the CT scans, dehiscence of the semicircular canals was defined by absence of high attenuation bone coverage in at least two planes, conversely it was indicated by the absence of low-signal bone margins on the MR images. ${ }^{24,25}$ The contrast 
between bone and soft tissue obtained by a bone dedicated window shows a perfect definition of bone structure.

According to a recent study, ${ }^{26}$ when a superior semicircular canal roof is evaluated by standard planes (axial, coronal, sagittal) in routine temporal CT images, falsepositive and false-negative diagnoses can be made. Indeed, creating the Pöschl plane increases the sensitivity and specificity of CT for diagnosis.

Several authors recommend that MR imaging (1.5T or 3T, T2-weighted, thickness slices $0.5 \mathrm{~mm}$ ) be conducted primarily because it does not contain ionizing radiation; moreover, with a sensitivity and negative predictive value of $100 \%$, it can conclusively exclude SSCD or PSCD. ${ }^{27,28}$

Surgical treatment of PSCD is rarely reported. We described the successful treatment of PSCD: jugular decompression and plugging of dehiscence. Usually, PSCD, due to a high jugular bulb, can be managed conservatively (watchful waiting), especially when there is hearing loss only: our patients experienced an important severity of symptoms due to misdiagnosis, including tinnitus, pressure-induced vertigo, autophony, causing significant disability. Finally, another aspect not to be underestimated is anxiety: it is a common comorbidity in many vestibular disorders, including SCD and should also be screened for and treated to the best extent possible. ${ }^{29}$

\section{Conclusion}

Bony dehiscence of the labyrinth causes an abnormal pressure gradient in the inner ear fluids, leading to pressure or sound induced vertigo ("third mobile window" phenomenon). PSCD is rarer than SSCD and symptoms are like other diseases, such as Menière disease.

Audiometric and vestibular examination are important even if diagnosis is impossible without radiological findings. CT has an important role both in the preoperative evaluation and postoperative follow-up.

Plugging without exposure of the dehiscent area has been a helpful and safe method for definitive treatment of this pathology. Furthermore, it is recommended intervening when the symptoms compromise with a normal life. SCD signs and symptoms in members of the same family are similar: the co-occurrence in twin sisters is a confirm of this concept. We believe our report contributes to evidence in support of a potential genetic basis for SCD even if additional genetic studies are needed to explain this condition.

\section{Ethics Statement}

The patients provided written informed consent for publication of their case reports and accompanying images. This study was conducted in accordance with the Declaration of Helsinki and was accepted by the Ethics Committee of Azienda Ospedaliera Universitaria Senese.

\section{Disclosure}

The authors report no conflicts of interest in this work.

\section{References}

1. Minor LB, Solomon D, Zinreich JS, Zee DS. Sound and/or pressureinduced vertigo due to bone dehiscence of the superior semicircular canal. Arch Otolaryngol. 1998;124(3):249-258. doi:10.1001/ archotol.124.3.249

2. May-Lan H. Third window lesions. Neuroimag Clin N Am. 2019;29 (1):57-92. doi:10.1016/j.nic.2018.09.005

3. Chien WW, Carey JP, Minor LB. Canal dehiscence. Curr Opin Neurol. 2011;24(1):25-31. doi:10.1097/WCO.0b013e328341ef88

4. Merchant SN, Rosowski JJ. Conductive hearing loss caused by thirdwindow lesions of the inner ear. Otol Neurotol. 2008;29(3):282-289. doi:10.1097/mao.0b013e318161ab24

5. Kaski D, Davies R, Luxon L, et al. The Tullio phenomenon: a neurologically neglected presentation. $J$ Neurol. 2012;259(1):4-21. doi:10.1007/s.00415-011-6130-x

6. Shuman AG, Rizvi SS, Pirouet CW, et al. Hennebert's sign in superior semicircular canal dehiscence syndrome: a video case report. Laryngoscope. 2012;122(2):412-414. doi:10.1002/lary.22413

7. Minor LB. Superior canal dehiscence syndrome. Am J Otol. 2000;21 (1):9-19. doi:10.1016/S0196-0709(00)80068-X

8. Minor LB, Carey JP, Cremer PD, et al. Dehiscence of bone overlying the superior canal as a cause of apparent conductive hearing loss. Otol Neurotol. 2003;24(2):270-278. doi:10.1097/00129492-20030300000023

9. Gopen Q, Zhou G, Poe D, et al. Posterior semicircular canal dehiscence: first reported case series. Otol Neurotol. 2010;31(2):339-344. doi:10.1097/MAO.0b013e3181be65a4

10. Krombach GA, Di Martino E, Schmitz-Rode T, et al. Posterior semicircular canal dehiscence: a morphologic cause of vertigo similar to superior semicircular canal dehiscence. EurRadiol. 2003;13 (6):1444-1450. doi:10.1007/s00330-003-1828-5

11. Spasic M, Trang A, Chung LK. Clinical characteristics of posterior and lateral semicircular canal dehiscence. J Neurol Surg B Skull Base. 2015;76(6):421-425. doi:10.1055/s-0035-1551667

12. Schutt CA, Kveton JF. Posterior semicircular canal dehiscence secondary to jugular enlargement. Am J Otolaryngol. 2016;37(3):173174. doi:10.1016/j.amjoto.2015.10.008

13. Park JJ, Shen A, Loberg C, Westhofen M. The relationship between jugular bulb position and jugular bulb related inner ear dehiscence: a retrospective analysis. Am J Otolaryngol. 2015;36(3):347-351. doi:10.1016/j.amjoto.2014.12.006

14. Ward BK, Carey JP, Minor LB. Superior canal dehiscence syndrome: lessons from the first 20 years. Front Neurol. 2017;8:177. doi:10.3389/fneur.2017.00177

15. Carey JP, Minor LB, Nager GT. Dehiscence or thinning of bone overlying the superior semicircular canal in a temporal bone survey. Arch Otolaryngol Head Neck Surg. 2000;126(2):137-147. doi:10.1001/archotol.126.2.137 
16. Hirvonen TP, Weg N, Zinreich SJ, Minor LB. High-resolution CT findings suggest a developmental abnormality underlying superior canal dehiscence syndrome. Acta Otolaryngol. 2003;123(4):477481. doi:10.1080/0036554021000028099

17. Minor LB. Clinical manifestations of superior semicircular canal dehiscence. Laryngoscope. 2005;115(10):1717-1727. doi:10.1097/ 01.mlg.0000178324.55729.b7

18. Lee JA, Liu YF, Nguyen SA, McRackan TR, Meyer TA, Rizk HG. Posterior semicircular canal dehiscence: case series and systematic review. Otol Neurotol. 2020;41(4):511-521. doi:10.1097/MAO.000 0000000002576

19. Erdogan N, Songu M, Akay E, et al. Posterior semicircular canal dehiscence in asymptomatic ears. Acta Otolaryngol. 2011;131(1):4 8. doi:10.1016/j.amjoto.2014.12.006

20. Niesten MEF, Lookabaugh S, Curtin H, et al. Familial superior canal dehiscence syndrome. JAMA Otolaryngol Head Neck Surg. 2014;140 (4):363-368. doi:10.1001/jamaoto.2013.6718

21. Heidenreich KD, Kileny PR, Ahmed S, et al. Superior canal dehiscence syndrome affecting 3 families. JAMA Otolaryngol Head Neck Surg. 2017;143(7):656-662. doi:10.1001/jamaoto.2016.4743

22. Schutt CA, Neubauer P, Samy RN, et al. The correlation between obesity, obstructive sleep apnea, and superior semicircular canal dehiscence: a new explanation for an increasingly common problem. Otol Neurotol. 2015;36(3):551-554. doi:10.1097/MAO.0000000000000555

23. Mikulec AA, McKenna MJ, Ramsey MJ, et al. Superior semicircular canal dehiscence presenting as conductive hearing loss without vertigo. Otol Neurotol. 2004;25(2):121-129. doi:10.1097/00129492200403000-00007
24. Cloutier JF, Bélair M, Saliba I. Superior semicircular canal dehiscence: positive predictive value of high-resolution CT scanning. Eur Arch Otorhinolaryngol. 2008;265(12):1455-1460. doi:10.1007/ s00405-008-0672-2

25. Stimmer H, Hamann KF, Zeiter S, et al. Semicircular canal dehiscence in HR multislice computed tomography: distribution, frequency, and clinical relevance. Eur Arch Otorhinolaryngol. 2012;269(2):475-480. doi:10.1007/s00405-011-1688-6

26. Duman IS, Dogan SN. Contribution of reformatted multislice temporal computed tomography images in the planes of stenvers and pöschl to the diagnosis of superior semicircular canal dehiscence. J Comput Assist Tomogr. 2020;44(1):53-58. doi:10.1097/RCT.0000000000000957

27. Browaeys P, Larson TL, Wong ML, Patel U. Can MRI replace CT in evaluating semicircular canal dehiscence? AJNR Am J Neuroradiol. 2013;34(7):1421-1427. doi:10.3174/ajnr.A3459

28. Inal M, Burulday V, Bayar Muluk N, et al. Magnetic resonance imaging and computed tomography for diagnosing semicircular canal dehiscence. J Craniomaxillofac Surg. 2016;44(8):998-1002. doi:10.1016/j.jcms.2016.06.006

29. Staab JP, Ruckenstein MJ. Which comes first? Psychogenic dizziness versus otogenic anxiety. Laryngoscope. 2003;113(10):1714-1718. doi:10.1097/00005537-200310000-00010

\section{Publish your work in this journal}

Reports in Medical Imaging is an international, peer-reviewed, open access journal publishing original research, reports, reviews and commentaries on all areas of medical imaging. The manuscript management system is completely online and includes a very quick and fair peerreview system, which is all easy to use. Visit http://www.dovepress. com/testimonials.php to read real quotes from published authors. 